\title{
二次元接合体に対するエンリッチ有限要素法の適用（対数特異性の場合） Application of an enriched FEM to 2D-dissimilar material joints (In case of power-logarithmic singularities model)
}

\author{
O Chonlada LUANGARPA, Graduate school of Nagaoka University of Technology 1603-1 Kamitomioka, Niigata \\ Hideo KOGUCHI, Nagaoka University of Technology 1603-1 Kamitomioka, Niigata
}

Key Words: Stress singularity, Enriched finite element method, Power-logarithmic singularitiy

\section{Introduction}

Dissimilar material joints have singularities created by discontinuities in material properties across an interface. For this reason, it is difficult to predict accurate stress by using conventional FEM. There are some special elements or methods developed for solving this problem. An enriched finite element method developed by Benzley ${ }^{(1)}$ is one method that do not need to divide meshes around the singular point to be very small meshes and can directly compute the intensity of singularity while conventional FEM hard to separate the intensity of singularities in case of multi-singularities.

In the present study, the singular stress field in three-material joints with power-logarithmic singularities is analyzed by an enriched finite element method. An eigenvalue and eigenvector analysis is applied to calculate the order of stress singularities and the asymptotic displacement fields on the enriched elements. Furthermore, enriched element size and area are varied to study its convergence and to find a suitable element for each case.

\section{Analytical formula}

2D singular stress field around the singular point with power-logarithmic stress singularities can be described by

$$
\sigma_{i j}=\bar{K}_{i j 1} r^{-\lambda} h_{i j 1}(\theta)+\bar{K}_{i j 2} r^{-\lambda}\left[-\ln (r) h_{i j 1}(\theta)+h_{i j 3}(\theta)\right]
$$

where $r$ is the radial distance from the singular point, $\lambda$ is the order of stress singularity, $\bar{K}_{i j k}$ and $h_{i j k}(\theta) ;(i, j=r$ or $\theta, k=1$, $2)$, are intensity of singularities and angular functions, respectively.

2D enriched element equations were developed by Benzley ${ }^{(1)}$ to solve an intensity in stress and displacement for two-dimensional singular point. In this method, three different elements; enriched, transition and standard elements, are used. (See Fig.1)

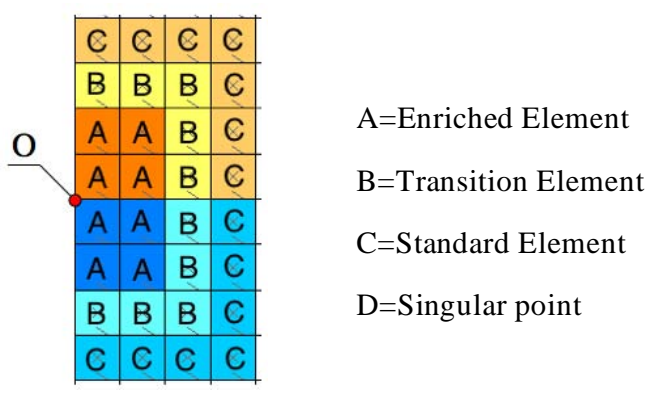

Fig. 1 Element models for Enriched Analysis
The displacement assumption for the enriched element is of form

$$
\begin{aligned}
& u_{k}=\sum_{n=1}^{m} g_{n} \bar{u}_{k n}+\bar{K}_{\theta \theta 1}\left(Q_{k 1}-\sum_{n=1}^{m} g_{n} \bar{Q}_{k 1}\right)+ \\
& \bar{K}_{\theta \theta 2}\left[-\ln (r) Q_{k 1}+Q_{k 3}-\sum_{n=1}^{m} g_{n}\left(-\ln (r) \bar{Q}_{k n 1}+\bar{Q}_{k n 3}\right)\right]
\end{aligned}
$$

In eq.(2), $u_{1}$ and $u_{2}$ represent the displacements of a point within the element in the $x$ - and $y$-directions, respectively. $\underline{Q}_{k 1}$ and $Q_{k 3}$ are the asymptotic displacement fields. $\bar{u}_{k n}$, $\bar{Q}_{k n 1}$ and $\bar{Q}_{k n 3}$ are the values of $u_{k}, Q_{k 1}(r, \theta)$ and $Q_{k 3}(r, \theta)$ evaluated at node $n, m$ is the number of node in an element and $g_{n}$ is the shape function in a standard finite element.

For transition elements (type B element), they are needed to join enriched elements to standard elements in a finite element model. The displacement field in a transition element is given by the relationship

$$
\begin{aligned}
& u_{k}=\sum_{n=1}^{m} g_{n} \bar{u}_{k n}+R(\xi, \eta)\left\{\bar{K}_{\theta \theta 1}\left(Q_{k 1}-\sum_{n=1}^{m} g_{n} \bar{Q}_{k 1}\right)+\right. \\
& \left.\bar{K}_{\theta \theta 2}\left[-\ln (r) Q_{k 1}+Q_{k 3}-\sum_{n=1}^{m} g_{n}\left(-\ln (r) \bar{Q}_{k n 1}+\bar{Q}_{k n 3}\right)\right]\right\}
\end{aligned}
$$

where $R(\xi, \eta)$ is a 'zeroing' function, equals to 1 along 'enrich' boundaries and equals to 0 along 'standard' boundaries.

\section{Numerical analysis}

3 - 1 The model for analysis

The model for analysis is shown in Fig 2. It is the three-material model fixed on the bottom side and applied shear stress on the top. In this analysis, the height of material 1 and material 2 are fixed at $10 \mathrm{~mm}\left(h_{1}=h_{2}=10 \mathrm{~mm}\right)$. The model length $L_{1}$ and $L_{2}$ are equally at $60 \mathrm{~mm}$ and shear loading on the top surface is $1 \mathrm{MPa}$. Material properties are shown in Table 1.

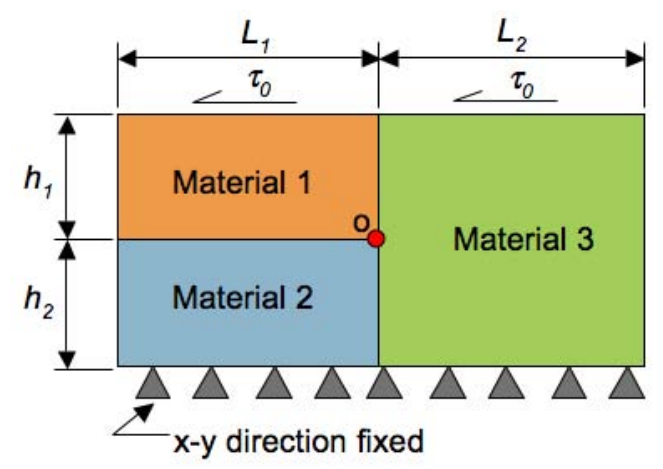

Fig. 2 Analytical model and boundary condition 
Table 1 Material properties

\begin{tabular}{|c|c|c|}
\hline & Young's modulus $(\mathrm{GPa})$ & Poisson's ratio \\
\hline Material 1 & 160.0 & 0.3 \\
\hline Material 2 & 4.0 & 0.3 \\
\hline Material 3 & 16.123 & 0.3 \\
\hline
\end{tabular}
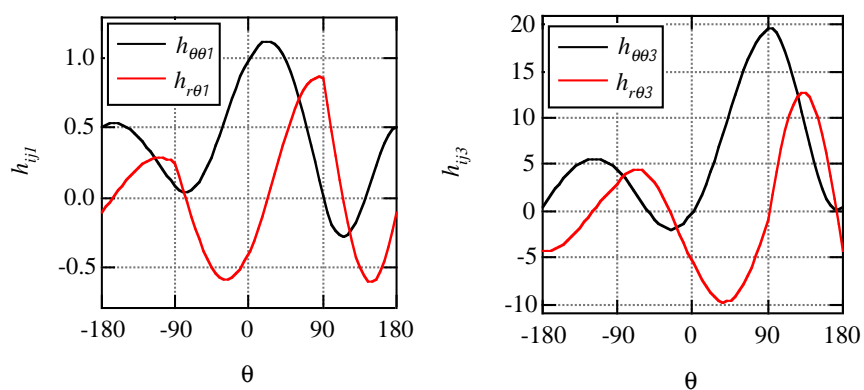

Fig. 3 Angular functions; $h_{i j 1}$ and $h_{i j 3}$ respectively

\section{2 Eigenvalue analysis}

Eigen analysis is used to find the asymptotic displacement fields in the enriched element. The eigen equation can be expressed as:

$$
\left(p^{2}[A]+p[B]+[C]\right)\{u\}=0
$$

where $[A],[B]$ and $[C]$ are matrices compose of Young's modulus and Poisson's ratio, $p=1-\lambda$ and $\{u\}$ is the eigenvector of displacement.

The results from eigen analysis show that there are 2 real-singularities which the orders of stress singularity $\left(\lambda_{1}=\right.$ 0.3474 and $\lambda_{2}=0.3466$ ) are very close together. Next step, eigenvector analysis is applied to evaluate the angular displacements and then converted to the angular functions; $h_{i j 1}(\theta)$ and $h_{i j 3}(\theta)$ in eq.(1), following stresses and displacements relationship. (See Fig. 3)

\section{3 Enriched FEM}

On this analysis, 4-node element is used for simplicity. The element model around the singular point is shown in Fig. 4. To study the influence of mesh refinement and the enriched area size on the result convergence, the size of enriched elements $(b)$ is changed from 0.05 to $1.0 \mathrm{~mm}$. and the enriched area $(a)$ is varied from 0.5 to $4.0 \mathrm{~mm}$.

The results of the intensity of singularities $\left(\bar{K}_{\theta \theta 1}\right.$ and $\left.\bar{K}_{\theta \theta 2}\right)$ with various enriched element sizes and various enriched areas are shown in Figs. 5-6. In this study, the results are compared with conventional FEM's results (analyzed by Marc program) which determine the intensity of singularities followed Munz and Yang ${ }^{(2)}$, s fitting technique $\left(K_{\theta \theta 1}=1.609\right.$ and $\left.K_{\theta \theta 2}=0.244\right)$.

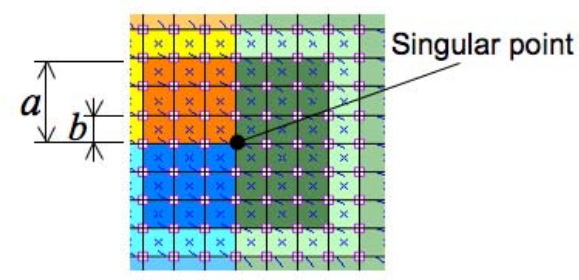

Fig. 4 Element model around the singular point
As can be seen on these figures, the intensity of singularities converges to the results in conventional FEM when the element size is smaller.

\section{Conclusions}

The results obtained applying the enriched FEM on power-logarithmic singularities model are agreed with those using conventional FEM. The accuracy of the results can be improved by using smaller size of the enriched elements.

\section{References}

(1) Benzley, S.E., Int. J. Numerical Methods in Engineering, 8, 537-545 (1974)

(2) D. Munz and Y.Y. Yang, Int. J. Fracture, 60, 169-177 (1993)

(3) Pageau S.S., Bigger S.B., Int. J. Numerical Methods in Engineering, 40, 2693-2713 (1997)

(4) Koguchi, H and Luangarpa, C., Journal of Solid Mechanics and Materials Engineering, Vol. 2, 319-332, (2008).

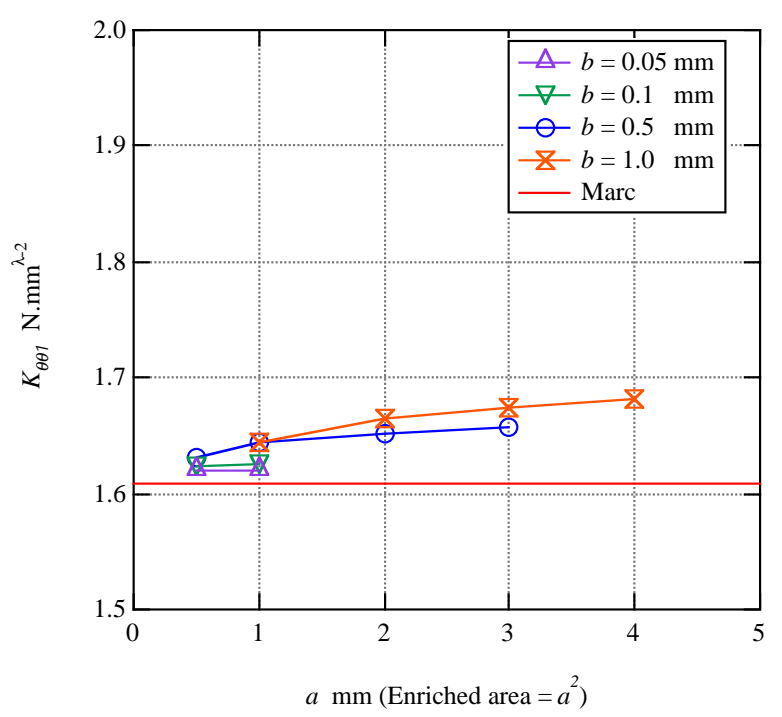

Fig. 5 The 1-intensity of singularity, $K_{\theta \theta 1}$, against enriched area

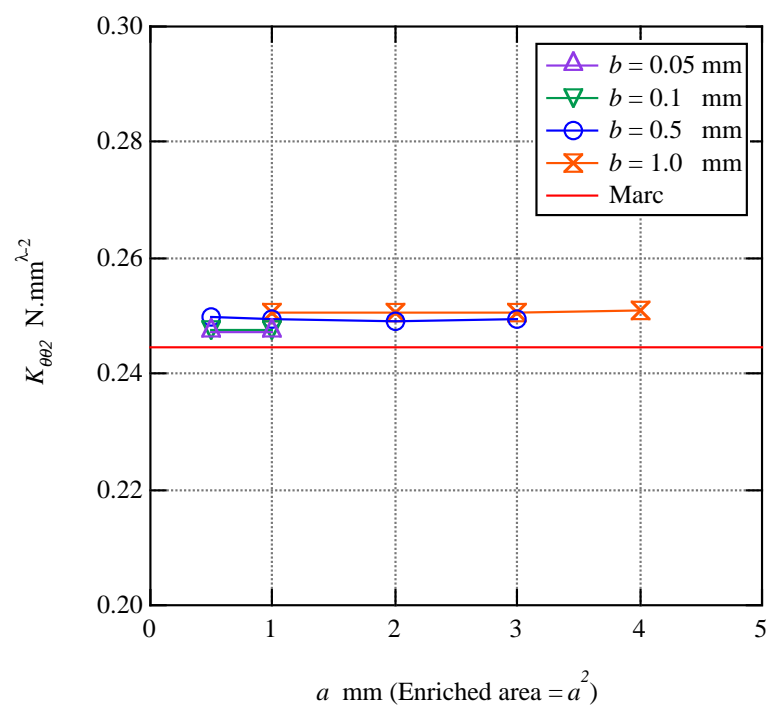

Fig. 6 The 2-intensity of singularity, $K_{\theta \theta 2}$, against enriched area 